\title{
Outcome of bacteremia and fungemia in paediatric oncology patients
}

\author{
Joan L Robinson MD¹, Robert P Rennie PhD DABMM²
}

JL Robinson, RP Rennie. Outcome of bacteremia and fungemia in paediatric oncology patients. Can J Infect Dis 2002;13(6):375-381.

OBJECTIVE: To determine the outcome of paediatric oncology patients with positive blood cultures.

DESIGN: Retrospective chart review.

SETTING: Tertiary care hospital.

POPULATION STUDIED: Oncology patients up to 17 years of age with positive blood cultures from January 1, 1994 to March 31, 1999.

MAIN RESULTS: There were 121 episodes of positive blood cultures in 76 patients. Seventeen episodes were excluded because blood cultures were contaminated. Of the organisms grown from the remaining episodes, 63\% were Gram-positive organisms, 23\% were Gram-negative organisms, 3\% were fungal and $11 \%$ were mixed. There were 13 episodes with pure or mixed isolates of Staphylococcus aureus, of which nine occurred within 14 days of the placement of a new central venous tunnelled catheter. Central venous tunnelled catheters were retained in 76 of the 102 episodes when they were present. There were two relapses, and four children were admitted to the intensive care unit with septic shock, but all survived.

CONCLUSIONS: The outcome was excellent with the current management of possible bacteremia in paediatric oncology patients, but the high incidence of $S$ aureus bacteremia suggests that empirical antibiotics should be altered if sepsis is suspected within 14 days of the placement of a central venous catheter.

\section{Bactériémie et fongémie en oncologie pédia- trique}

OBJECTIF : Déterminer l'évolution des patients en oncologie pédiatrique qui présentent des hémocultures positives.

PLAN D'ÉTUDE : Examen rétrospectif de dossiers.

MILIEU : Hôpital de soins tertiaires.

POPULATION À L'ÉTUDE : Patients en oncologie âgés de 17 ans et moins, qui ont produit des hémocultures positives et qui ont été traités

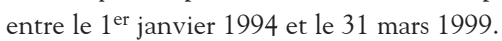

PRINCIPAUX RÉSULTATS : Nous avons relevé 121 épisodes d'hémoculture positive chez 76 patients, dont 17 ont été exclus pour cause de contamination. Des épisodes restants, $63 \%$ étaient à germes Gram positif; $23 \%$, à germes Gram négatif; $3 \%$, à champignons; $11 \%$ étaient mixtes. Treize cultures étaient constituées d'isolats purs ou mixtes de Staphylococcus aureus (SA), dont neuf ont été réalisées dans les 14 jours suivant la pose d'un nouveau cathéter veineux central tunnellisé. Dans 76 épisodes sur 102, les cathéters veineux centraux tunnellisés ont été maintenus en place. Il y a eu deux rechutes, et quatre enfants ont été admis à l'unité des soins intensifs en raison d'un choc septique, mais ils ont tous survécu.

CONCLUSION : Le traitement actuel des bactériémies possibles donne d'excellents résultats en oncologie pédiatrique, mais la forte incidence de bactériémie à SA laisse penser qu'il faudrait modifier le traitement empirique aux antibiotiques si l'on soupçonne la présence d'une septicémie dans les 14 jours suivant la pose d'un cathéter veineux central.

Key Words: Bacteremia; Empirical antibiotics; Fungemia; Oncology; Paediatric

Departments of ${ }^{1}$ Pediatrics and ${ }^{2}$ Medical Microbiology and Immunology, University of Alberta, Edmonton, Alberta

Correspondence: Dr Joan L Robinson, 2C3 Walter Mackenzie Centre, University of Alberta, Edmonton, Alberta T6G $2 B 7$.

Telephone 780-407-1680, fax 780-407-7136, e-mail jr3@ualberta.ca

Received for publication September 21, 2001. Accepted December 28, 2001 
$\mathrm{B}$ acteremia and fungemia are common causes of morbidity in paediatric oncology patients. Mucositis of the gastrointestinal tract undoubtedly results in increased opportunities for bacteremia to occur. Impaired host defenses and the seeding of indwelling venous lines increase the chance that this bacteremia will be continuous rather than transient. Bacteremia also occurs following breaks in sterile technique during the manipulation of venous lines. The pathogenesis of fungemia in this patient population is not well delineated, but one likely source is from venous catheters being infected following transient fungemia from gut flora.

It is standard practice to start empirical antibiotics in all febrile oncology patients who are neutropenic. The empirical antibiotic regimen used in paediatric oncology patients in our centre is tobramycin and piperacillin. Most nonneutropenic febrile oncology patients are also admitted and started on these same antibiotics, because there is evidence that their incidence of bacteremia may be at least as frequent as that of neutropenic patients (1). In our centre, venous catheters are removed in the face of suspected line infection only if the venous catheters are nontunnelled, permanent venous access is no longer required, the infecting organism is a yeast, bacteremia persists after several days of appropriate antibiotics or the patient has septic shock that is thought to be line-related. The purpose of the present study was to evaluate the outcome of patients by using this approach to suspected bacteremia or fungemia.

\section{METHODS}

Using records from the University of Alberta Hospital microbiology laboratory, a retrospective review was done of all positive blood cultures received from paediatric oncology patients (up to 17 years of age) admitted to the oncology ward of the Stollery Children's Health Centre in Edmonton from January 1, 1994 to March 31, 1999. The blood culture protocol specified that 1 to $3 \mathrm{~mL}$ of blood be inoculated into a BACTEC Peds Plus vial (Becton Dickinson, USA), and isolates were recovered with the use of the BACTEC 9240 system (Becton Dickinson, USA). The vast majority of blood cultures were drawn from venous catheters.

Blood culture isolates were identified in the University of Alberta Hospital microbiology laboratory by automated (Vitek, Biomereuix Ltd, USA) or standard reference level laboratory methods. Antimicrobial susceptibility testing for most isolates was performed by Vitek, or using standardized NCCLS reference methodologies.

The information collected at a chart review included the sex of the patient, the type of malignancy, antimicrobial susceptibilities of the blood culture isolate, the duration of antimicrobial therapy, the evidence of infection at another site with the same organism and the type of venous catheter in place at the time of the positive blood culture (peripheral, Broviac [CR Bard, USA] or implanted port). The duration of the placement of the venous catheter was recorded, and infections occurring within 14 days of the insertion of a catheter were considered to be possible surgical infections.
The standard definition of a surgical site infection is one that occurs within 30 days, but it seems likely that organisms introduced at the time of catheter insertion would result in bacteremia within 14 days.

To determine outcome, data were also recorded on the need for the removal of venous catheters for any reason during the time the patient was being treated for the positive blood culture. The results of all subsequent blood cultures and the details of any intensive care unit (ICU) admissions during the admission when the blood cultures were positive were recorded. A recurrence of positive blood cultures with the same organism was defined as a relapse if it occurred within 30 days of the child completing antimicrobial therapy for the positive blood culture.

An organism was considered to be a definite contaminant if bacteremia or fungemia resolved without appropriate antibiotics or venous catheter removal, and did not recur within 30 days. An episode was considered to be a possible bacteremia or fungemia if only a single blood culture was positive for an organism other than Staphylococcus aureus, Enterococcus species, nonviridans streptococci, Pseudomonas, Enterobacteriaceae, or yeast, and an antibiotic that could cover that organism was instituted before a repeat blood culture was obtained; more than one blood culture was positive for coagulase-negative staphylococci (CNS), but there were also multiple negative intercurrent blood cultures; or more than one blood culture was positive for CNS, but the patient had no fever or hypotension within $24 \mathrm{~h}$ of these cultures being drawn. All other blood culture isolates were considered to represent definite bacteremia or fungemia. Cultures with mixed growth were classified as definite bacteremia or fungemia if any one of the organisms grown was $S$ aureus, Enterococcus species, nonviridans streptococci, Pseudomonas, Enterobacteriaceae or yeast, or if at least one of the other organisms was isolated from more than one blood culture and the patient had fever or hypotension within $24 \mathrm{~h}$ of that culture being drawn.

Multiple positive cultures for the same organism were considered to be a single episode of positive blood cultures unless they occurred more than 30 days apart. If a different organism grew from a patient at any time after the initial positive blood culture had cleared, this was considered to be a separate episode of bacteremia or fungemia. If the isolate from the initial positive blood culture had not yet cleared, this was considered to be the same episode.

\section{RESULTS}

Episodes of positive blood cultures

There were 121 episodes of positive blood cultures in 76 patients (37 male, 39 female). In 17 episodes (8\%), the organism (CNS in every case) grew from a single blood culture and resolved without appropriate antibiotics. These episodes were thought to represent contamination rather than bacteremia. There were 45 episodes of positive blood cultures that fit our definition of possible bacteremia or fungemia. The remaining 59 episodes were thought to rep- 
TABLE 1

The organisms grown from 104 episodes of bactermia and/or fungemia in paediatric oncology patients, 1994 to 1999

\begin{tabular}{|c|c|c|c|}
\hline Organism & & Number of episodes & \\
\hline & Definite & Possible & Resolved without \\
\hline Gram-positive bacteria & bacteremia & bacteremia & line removal \\
\hline Staphylococcus aureus & 11 & & 6 \\
\hline Streptococcus pneumoniae & 3 & & 3 \\
\hline Enterococcus species & 2 & & 1 \\
\hline Group G Streptococcus & 1 & & $0^{*}$ \\
\hline Coagulase negative staphylococci (CNS) & & & \\
\hline Viridans group streptococci (VGS) & 15 & 19 & 26 \\
\hline Diphtheroids & 2 & 9 & 9 \\
\hline Micrococcus species & & 1 & 0 \\
\hline Aerobic spore forming bacillus & & 1 & 1 \\
\hline Gram-negative bacteria & & & \\
\hline Escherichia coli & 6 & & $3^{*}$ \\
\hline Klebsiella pneumoniae & 4 & & 4 \\
\hline Enterobacter cloacae & 3 & & 2 \\
\hline Salmonella enteritidis & 1 & & 1 \\
\hline Capnocytophaga species & & 3 & 3 \\
\hline Pasteurella multocida & & 1 & 1 \\
\hline Moraxella catarrhalis & & 1 & 1 \\
\hline Moraxella osloensis & & 1 & 1 \\
\hline Neisseria elongata & & 1 & 1 \\
\hline Aerobic Gram-negative cocci & & 1 & 1 \\
\hline Agrobacterium radiobacter & & 2 & 1 \\
\hline & Definite & Possible & Resolved without \\
\hline Fungi & fungemia & fungemia & line removal \\
\hline Candida tropicalis & 2 & & 0 \\
\hline Candida albicans & 1 & & 0 \\
\hline Mixed organisms & $\begin{array}{c}\text { Definite bacteremia/ } \\
\text { fungemia }\end{array}$ & $\begin{array}{c}\text { Possible bacteremia/ } \\
\text { fungemia }\end{array}$ & $\begin{array}{l}\text { Resolved without } \\
\text { line removal }\end{array}$ \\
\hline Group B Streptococcus aureus & 2 & & 1 \\
\hline Escherichia coli, Citrobacter freundii & 1 & & 1 \\
\hline Klebsiella pneumoniae, Pseudomonas aeruginosa & 1 & & 1 \\
\hline CNS, Acinetobacter, Pseudomonas aeruginosa & 1 & & 1 \\
\hline CNS, Comamonas, Agrobacterium tumefaciens & 1 & & 0 \\
\hline Stomatococcus, CNS, VGS & 1 & & 1 \\
\hline Enterobacter cloacae, Klebsiella pneumoniae & 1 & & 1 \\
\hline Lactobacillus, Candidas albicans & 1 & & 0 \\
\hline Aspergillus fumigatus, Aspergillus versicolor & & 1 & 1 \\
\hline Stenorophomonas maltophilia, Leclercia adecarboxylata & & 1 & 1 \\
\hline Stenotrophomonas maltophilia, CNS & & 1 & 0 \\
\hline
\end{tabular}

*In the one case with group $G$ Streptococcus and in one of the cases with Escherichia coli, the line that was removed was a peripheral line. All other lines that were removed were tunnelled central venous lines

resent definite bacteremia or fungemia. The organisms isolated from the episodes classified as possible or definite bacteremia or fungemia are shown in Table 1; 63\% were Gram-positive, 23\% were Gram-negative, 3\% were fungal and $11 \%$ had mixed organisms. Nine of the 11 viridans group streptococci (VGS) had reduced susceptibility to penicillin. All pneumococci were susceptible to penicillin, all $S$ aureus were susceptible to cloxacillin, and both ente- 


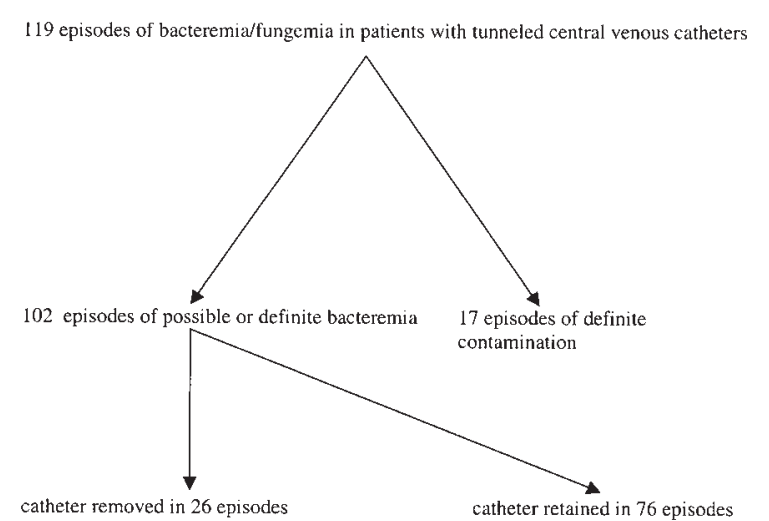

Figure 1) Outcome of 119 episodes of bacteremia in paediatric oncology patients with central venous tunnelled catheters (another two episodes occurred in patients without a central venous tunnelled catheter). Four episodes occurred in patients with implanted ports and 117 episodes in patients with Broviacs (CR Bard Inc, USA). All the catheters that were removed were Broviacs except one implanted port in a patient with fungemia

rococci were susceptible to ampicillin with gentamicin synergy. All coliforms and Pseudomonas isolates were susceptible to both gentamicin and tobramycin except for one isolate of Escherichia coli, which was susceptible to ampicillin and tobramycin, but resistant to gentamicin.

\section{Duration of antimicrobial therapy}

Appropriate antibiotics were continued for six to 42 days (median 10 days) in patients with possible or definite bacteremia. In cases where the duration of bacteremia could be determined and the venous catheter was not removed, the bacteremia resolved within three days of appropriate antibiotics being started, with the exception of four cases. These included two cases where bacteremia with CNS persisted for four days and one case where it persisted for five days, yet was ultimately cleared with vancomycin. In the fourth case, blood cultures from an implanted port were positive for Klebsiella pneumoniae, but were negative by the second day of tobramycin and cefuroxime. The patient's treatment was then changed to cefazolin, and blood cultures were positive on days 6 through 12. Cefazolin was switched to cefotaxime on day 8. Although the isolate was initially susceptible to cefazolin, it later had only intermediate susceptibility. The bacteremia did not recur.

\section{Outcome of central venous tunnelled catheters}

All of the episodes of positive blood cultures occurred in patients with a central venous tunnelled catheter, except for two episodes in patients with peripheral venous access (one with $E$ coli and one with group $G$ beta-hemolytic streptococcus [GBS] bacteremia). The outcome of the oth- er 119 episodes of bacteremia is shown in Figure 1, with $25 \%$ of patients with possible or definite bacteremia or fungemia having their central venous tunnelled catheter removed. Table 1 shows the episodes that resolved without the removal of a venous line. The reasons for catheter removal are outlined in Table 2; 11 of the 26 catheter removals occurred because of failure to clear bacteremia or fungemia, and four occurred because of other suspected manifestations of persistent infection. In the remaining 11 cases, the catheter was removed because it was not functioning well or was no longer required. Bacteremia or fungemia always resolved promptly with venous catheter removal and there was never evidence of infection at another site after the catheter had been removed. When required, tunnelled venous catheters were replaced two to 12 days after removal, with no recurrences of bacteremia or fungemia with the same organism.

\section{Etiology of early bacteremia after the placement of venous lines}

Of the 31 episodes of positive blood cultures that occurred in the first 14 days after the insertion of a central venous tunnelled catheter, two of the episodes were with contaminants. The isolates from the remaining episodes are shown in Table 3, with nine of the 29 episodes involving $S$ aureus.

\section{Relapses and recurrences of positive blood cultures}

There were only two relapses: a case with E coli (Table 3); and a case with mixed growth of CNS, Stomatococcus mucilaginosus, and VGS (where vancomycin was given on days 2 through 11, yet CNS grew again on day 14 and day 18 , and was ultimately cleared without the removal of the venous catheter by nine more days of vancomycin, starting on day 18). There were three cases of late recurrences with the same organism (CNS after seven and 11 months, and VGS after four months).

\section{ICU admissions}

During four of the episodes of bacteremia, the patient required ICU care because of septic shock (two episodes with pneumococci, one episode with $S$ aureus, and one episode with mixed growth of Enterobacter cloacae and K pneumoniae). The venous catheter was removed only in the patient with $S$ aureus, because the other infections were not clearly catheter-related. Two other bacteremic patients were in the ICU for problems that were unrelated to sepsis. There were no deaths attributed to sepsis.

\section{DISCUSSION}

The treatment outcome of bacteremia or fungemia in paediatric oncology patients was excellent in 121 episodes of positive blood cultures, using empirical tobramycin and piperacillin and venous catheter removal only in selected circumstances. There were no deaths and only four ICU admissions due to sepsis.

The isolated organisms are comparable with those reported in other studies of positive blood cultures in simi- 
TABLE 2

Reasons for the removal of a tunnelled venous catheter in 26 of 119 episodes of positive blood cultures

\begin{tabular}{|c|c|c|}
\hline Reason for catheter removal & $\begin{array}{l}\text { Number } \\
\text { of cases }\end{array}$ & Organism(s) \\
\hline Patient toxic & 2 & Case 1 and 2 - Staphylococcus aureus \\
\hline Ongoing embolic disease despite resolution of bacteremia & 1 & Staphylococcus aureus \\
\hline Suspected septic arthritis of the knee despite resolution of bacteremia & 1 & Staphylococcus aureus \\
\hline $\begin{array}{l}\text { Persistent cellulitis or pus at venous catheter site } \\
\text { despite resolution of bacteremia }\end{array}$ & 2 & $\begin{array}{l}\text { Case } 1 \text { - Enterobacter cloacae followed by } \\
\text { Micrococcus: case } 2 \text { - CNS, Commamonas, } \\
\text { Agrobacterium radiobacter }\end{array}$ \\
\hline Persistent bacteremia and pus at venous catheter site & 1 & Staphylococcus aureus and GBS \\
\hline $\begin{array}{l}\text { Recurrent bacteremia } 17 \text { days after a } 10 \text { day course } \\
\text { of antibiotics in a patient with a perianal ulcer } \\
\text { growing same organism }\end{array}$ & 1 & Escherichia coli \\
\hline $\begin{array}{l}\text { Failure to sterilize blood cultures despite at least } \\
\text { four days of appropriate antibiotics }\end{array}$ & 4 & $\begin{array}{l}\text { Case } 1 \text { and } 2 \text { - CNS: case } 3 \text { - Lactobacillus } \\
\text { then started growing Candida albicans day } 6 \text { : } \\
\text { case } 4-\text { Enterococcus species }\end{array}$ \\
\hline Fungemia - no attempt to clear with antifungals & 3 & \\
\hline Catheter no longer required & 7 & \\
\hline Mechanical problems with catheter & 4 & \\
\hline
\end{tabular}

CNS Coagulase-negative staphylococci; GBS Group G beta-hemolytic streptococcus

TABLE 3

Organisms grown from 29 episodes of bacteremia and/or fungemia in paediatric oncology patients within 14 days of placement of a central venous catheter

\begin{tabular}{|c|c|c|}
\hline Organism & $\begin{array}{c}\text { Definite } \\
\text { bacteremia/fungemia }\end{array}$ & Number of episodes \\
\hline Staphylococcus aureus & 8 & \\
\hline Coagulase negative staphylococci & & 4 \\
\hline Viridans group streptococci & 1 & 4 \\
\hline Escherichia coli & 2 & \\
\hline Klebsiella pneumoniae & 1 & \\
\hline Enterobacter cloacae & 1 & \\
\hline Capnocytophaga species & & 3 \\
\hline Pasteurella multocida & & 1 \\
\hline Neisseria elongata & & 1 \\
\hline Candida tropicalis & 1 & \\
\hline Group B Streptococcus, Staphylococcus aureus & 1 & \\
\hline Klebsiella pneumoniae, Pseudomonas aeruginosa & 1 & \\
\hline
\end{tabular}

lar patient populations, except for the high frequency of $S$ aureus (accounting for $22 \%$ of definite bacteremias in our study compared with less than $5 \%$ in other studies) $(2-4)$. Two of the episodes of $S$ aureus bacteremia occurred four days apart, but the other episodes were scattered throughout the years of the study. This makes it unlikely that the episodes had a common source. The odds ratio for the development of $S$ aureus bacteremia (including all cases of possible or definite bacteremia) in the first 14 days, compared with more than 14 days after the placement of a cen- 
tral venous catheter, was 5.66 (95\% CI 1.42 to 26.73 ). It seems likely that some of these infections were introduced when the venous lines were placed.

Although the outcome was very good with the use of tobramycin and piperacillin, in vitro susceptibilities of the blood culture isolates suggest that gentamicin would have worked as well as tobramycin, because the only coliform that was resistant was susceptible to piperacillin. Susceptibilities of the organisms received from all patients by our laboratory from July 1, 1999 to June 30, 2000 showed that all Enterobacteriaciae were susceptible to gentamicin, with the exception of $8 \%$ of Proteus mirabilis, $4 \%$ of E coli, $3 \%$ of Serratia marcescens, $2 \%$ of $K$ pneumoniae, $1 \%$ of Citrobacter freundii and $1 \%$ of E cloacae. Twenty-six percent of Pseudomonas aeruginosa were not susceptible to gentamicin. Despite the fact the coliforms and Pseudomonas isolates from our study and in our centre are usually susceptible to gentamicin, bacteremia with a gentamicin-resistant coliform or Pseudomonas could be rapidly fatal, so it would seem to be reasonable to continue to use tobramycin as empirical therapy. The substitution of cloxacillin for piperacillin may have been of benefit in the 13 episodes of $S$ aureus bacteremia (two of which were mixed with GBS). However, multiple other isolates may have responded better to piperacillin than to cloxacillin (including enterococci, Moraxella species, Neisseria species and anaerobes that may be present but do not grow in the blood culture vials). Because nine of the 13 episodes of $S$ aureus bacteremia occurred within 14 days of the placement of a new venous catheter, the addition of cloxacillin to the current empirical regimen, if the patient had had a tunnelled venous catheter inserted in the previous 14 days and has new onset of fever, should be considered in our centre. Although there was no mortality with waiting until the blood culture was growing Gram-positive organisms to start an antistaphylococcal drug, prolonged $S$ aureus bacteremia increases the risk of dissemination to other sites. Another option would be to substitute piperacillin-tazobactam for piperacillin in the current regimen to cover $S$ aureus, but widespread use of such a broad-spectrum agent may increase the incidence of antibiotic-resistant organisms. However, it would be a reasonable choice in the small percentage of patients who develop septic shock, in whom the risk of bacteremia with $S$ aureus, multiresistant coliforms or anaerobes is probably higher than in other patients.

Although CNS is the most common cause of bacteremia in paediatric oncology patients, and caused $34 \%$ of the episodes of possible or definite bacteremia or fungemia in our study, empirical vancomycin is not used in our centre. Studies done in adults have shown that it does not decrease morbidity in febrile neutropenic patients (5). Our study cannot address this issue, because it was retrospective and included patients who were not neutropenic. It is possible that the two patients who required catheter removal because of CNS bacteremia would have cleared their bacteremia if vancomycin was started initially rather than on day 2 of bacteremia. However, even if this were the case, it would not seem reasonable to have started vancomycin empirically in all 121 episodes of bacteremia or fungemia, and all the other febrile episodes with no positive blood cultures, to salvage two catheters.

From our study, 10 days of parenteral antimicrobial therapy appears to be adequate if bacteremia clears within $48 \mathrm{~h}$ and the venous catheter appears to be the likely source, or if the venous catheter is removed. A shorter course may be adequate in either situation, but this would require further study. Recently published Infectious Diseases Society of America guidelines recommend a five to seven day course for CNS if the catheter is removed, and a seven day course if the line is retained. They recommend a 10 to 14 day course for other organisms, assuming that the patient responds quickly to therapy and is not immunocompromised, but make no specific recommendations for the immunocompromised patient (6). However, in addition to parenteral antibiotics, they recommend a 14 day course of antibiotic lock therapy (instilling antibiotic combined with heparin into the lumen of the catheter). We have not used antibiotic lock therapy because there have been no randomized trials of its efficacy. It is also not clear if it should be used routinely (as recommended in the Infectious Diseases Society of America guidelines), or reserved as salvage therapy when parenteral antibiotics fail.

The vast majority of patients were cured without the removal of a central venous tunnelled catheter. In the cases in which these catheters were retained, only two bacteremias recurred within 30 days after antibiotics were stopped, and one of these was cured without the removal of the catheter. Only one of the four patients who was admitted to ICU with sepsis while bacteremic ultimately required venous catheter removal. The replacement of venous catheters removed because of infection appears to be safe once the bacteremia or fungemia clears.

However, four of the 11 episodes of $S$ aureus bacteremia and one of the two episodes with $S$ aureus and GBS bacteremia ultimately resulted in venous catheter removal, because of suspected infection of the catheter. Four of these five episodes requiring catheter removal occurred within 14 days of placement of the catheter. Because of the high risk of septic shock and disseminated infection from $S$ aureus bacteremia, and the high chance that bacteremia will not resolve with the catheter in place, some authors advise the removal of venous catheters in $S$ aureus bacteremia unless another source of bacteremia is evident (6). However, the use of transesophageal echocardiography to diagnose endocarditis and the use of antibiotic lock therapy may improve the outcome of $S$ aureus catheter-related infections (7). We would advocate the routine removal of the catheter if an infection occurs shortly after the catheter has been placed, and removal of older catheters if the bacteremia does not resolve within $24 \mathrm{~h}$ of starting the appropriate antimicrobial therapy.

The incidence of septic shock in paediatric oncology patients has not been well studied. Our ICU admission rate of $4 \%$ in patients with possible or definite bacteremia or 
fungemia compares favourably with a similar study that had an ICU admission rate of approximately 10\% (4).

\section{CONCLUSIONS}

The treatment outcome of paediatric oncology patients with suspected infection is excellent in our centre with empirical piperacillin and tobramycin, and the removal of venous catheters only in selected circumstances. However, the incidence of $S$ aureus infection is high in the first 14 days after placement of a new tunnelled venous catheter, so it may be worthwhile adding cloxacillin in patients who are febrile shortly after placement of a new venous catheter.

\section{REFERENCES}

1. Gorelick MH, Owen WC, Seibel NL, Reaman GH. Lack of association between neutropenia and the incidence of bacteremia associated with indwelling central venous catheters in febrile pediatric cancer patients. Pediatr Infect Dis J 1991;10:506-10.

2. Aquino VM, Pappo A, Buchanan GR, Tkaczewski I, Mustafa MM. The changing epidemiology of bacteremia in neutropenic children with cancer. Pediatr Infect Dis J 1995;14:140-3.

3. Harms D, Gortitz I, Lambrecht W, Kabisch H, Erttmannn R, Janka-Schaub G. Infectious risks of Broviac catheters in children with neoplastic diseases: A matched pairs analysis. Pediatr Infect Dis J 1992;11:1014-8.

4. Aledo A, Heller G, Ren L, et al. Septicemia and septic shock in pediatric patients: 140 consecutive cases on a pediatric hematology-oncology service. J Pediatr Hematol Oncol 1998;20:215-21.

5. Feld R. Vancomycin as part of initial empirical antibiotic therapy for febrile neutropenia in patients with cancer: Pros and cons. Clin Infect Dis 1999;29:503-7.

6. Mermel LA, Farr BM, Sheretz RJ, et al. Guidelines for the management of intravascular catheter-related infections. Clin Infect Dis 2001;32:1249-72.

7. Dudgale DC, Ramsey PG. Staphylococcus aureus bacteremia in patients with Hickman catheters. Am J Med 1990;89:137-41. 


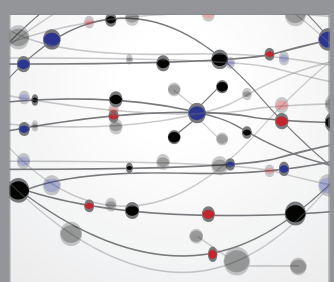

The Scientific World Journal
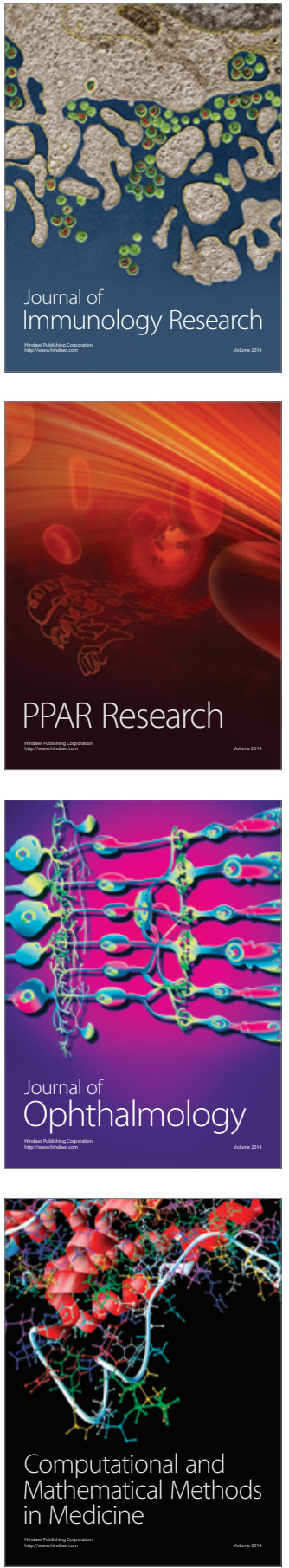

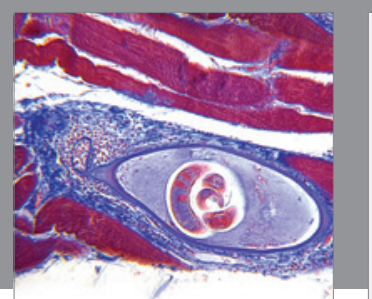

Gastroenterology Research and Practice

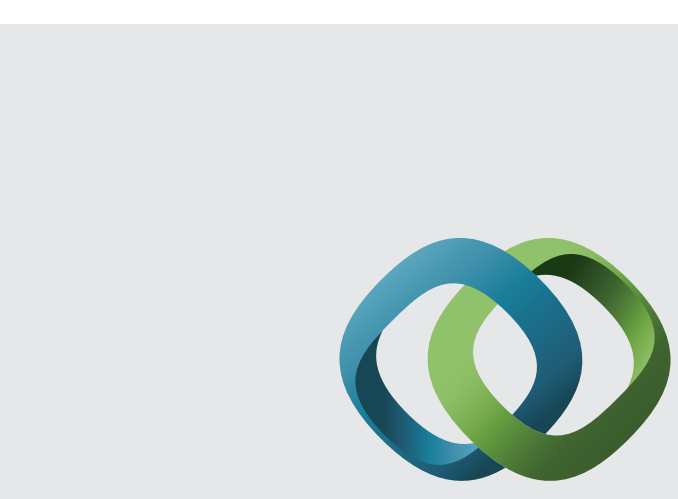

\section{Hindawi}

Submit your manuscripts at

http://www.hindawi.com
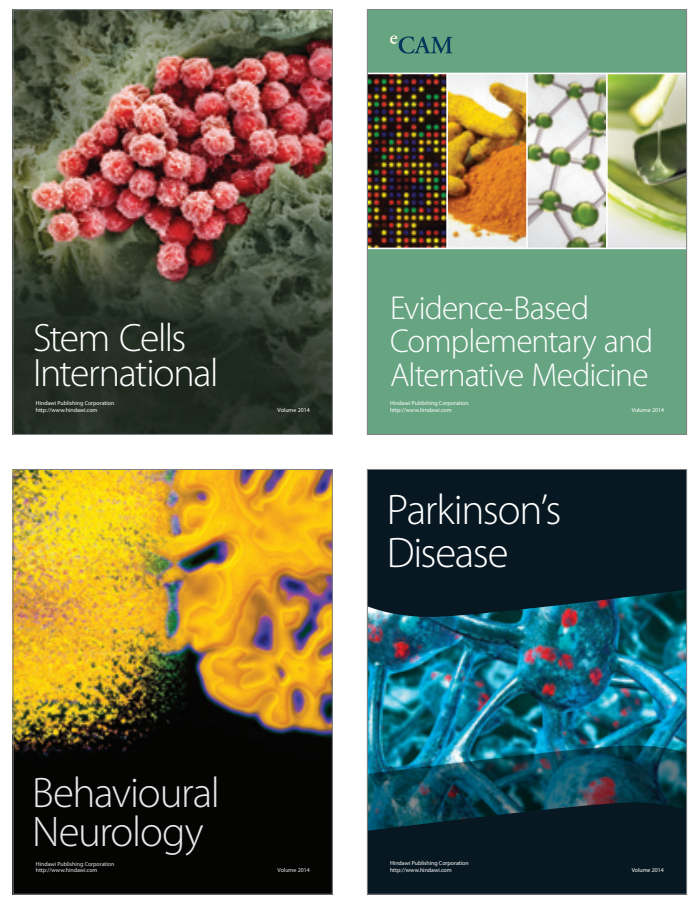
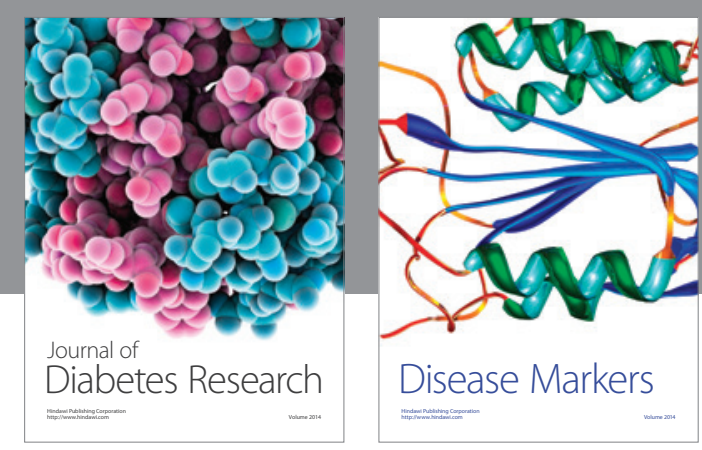

Disease Markers
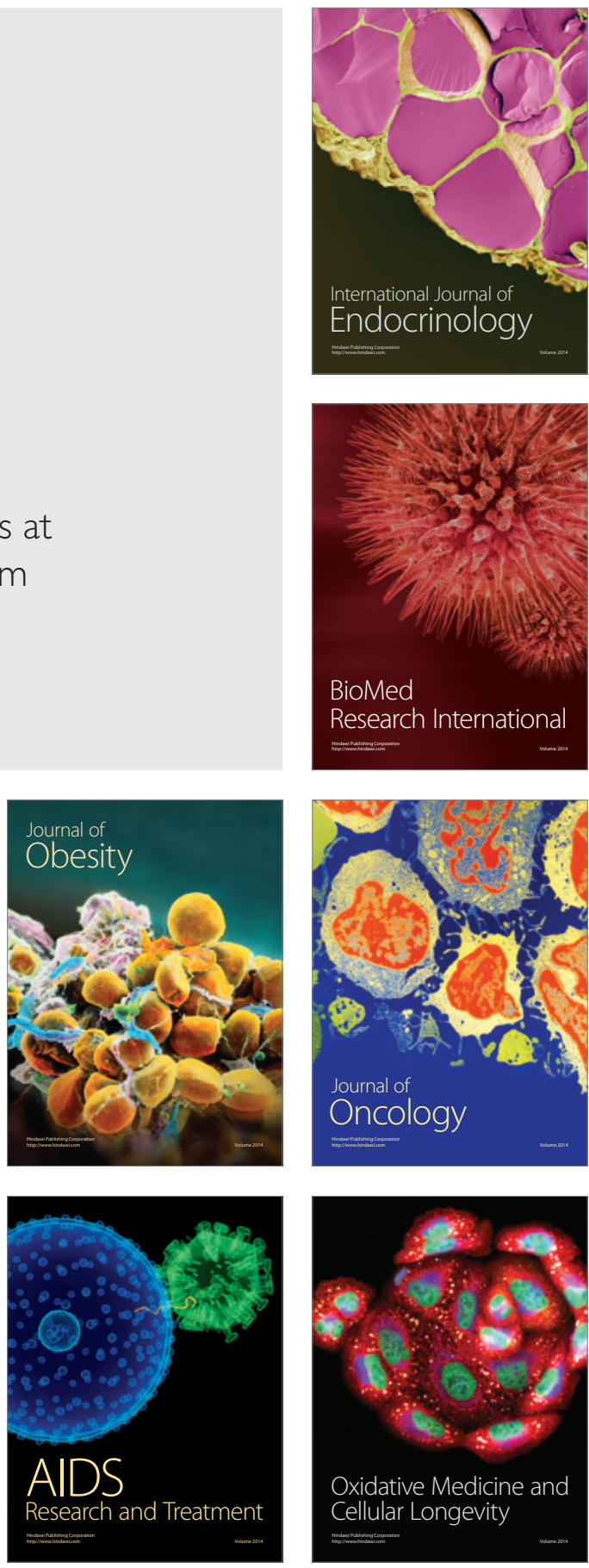